\title{
Disease Burden and Treatment Patterns of Psoriasis in Russia: A Real-World Patient and Dermatologist Survey
}

\author{
Alexey A. Kubanov - Andrey L. Bakulev - Tatiana V. Fitileva • \\ Evgenia Novoderezhkina · Isabelle Gilloteau • Haijun Tian • \\ Tanya Howe · Guilhem Pietri
}

Received: July 16, 2018 / Published online: September 19, 2018

(C) The Author(s) 2018

\section{ABSTRACT}

Introduction: Data regarding disease burden and quality of life (QoL) for patients with psoriasis from Russia are limited. The objective of this study was to describe the demographic and clinical characteristics, comorbidities, and treatment patterns of systemic therapy eligible psoriasis patients in Russia in order to assess the

Enhanced digital features To view enhanced digital features for this article go to https://doi.org/10.6084/ m9.figshare.7058996.

A. A. Kubanov

State Research Centre of Dermatology and

Venerology of the Russian Ministry of Health,

Moscow, Russia

A. L. Bakulev ( $\square)$

State Medical University of the Ministry of Health, Saratov, Russia

e-mail:al_ba05@mail.ru

T. V. Fitileva $\cdot$ E. Novoderezhkina

Novartis Pharma LLC, Moscow, Russia

I. Gilloteau

Novartis Pharma AG, Basel, Switzerland

H. Tian

Novartis Pharmaceuticals Corporation, East

Hanover, NJ, USA

T. Howe

GfK, London, UK

G. Pietri

Data Pyxis Ltd., St Albans, UK impact of psoriasis on the QoL and work productivity of the patients and to evaluate patient/dermatologist concordance on disease severity, signs/symptoms, and satisfaction with psoriasis treatment.

Methods: Data were collected by the Growth from Knowledge Disease Atlas global real-world evidence programme from nine countries. The data from the Russian population are presented here. Adult patients who had a current or prior history of moderate-to-severe psoriasis and were receiving prescription treatment at the time of the survey were included. Dermatologist-reported data on disease severity, symptoms, comorbidities, and treatment as well as patientreported data on QoL and work productivity were collected. Descriptive analysis of the data was conducted. Patient/dermatologist concordance was assessed using Cohen's $\kappa$.

Results: A total of 300 patients from Russia were included. The mean Psoriasis Area and Severity Index score was 9.0 and the mean disease duration was 9.9 years. The proportion of patients with itch, skin pain, and comorbidities increased as current psoriasis severity increased. The disease had a negative impact on patients' QoL (mean Dermatology Life Quality Index score: 7.1$)$ and work productivity (33.2\% drop in work productivity), which further deteriorated as disease severity increased. A large proportion of the enrolled patients (60\%) were treated with topical agents only. Overall, the level of concordance between patients and their 
dermatologists regarding psoriasis severity and satisfaction with overall disease control achieved was low.

Conclusion: Results demonstrate a substantial disease burden on psoriasis patients in Russia, despite receiving treatment for their psoriasis, as well as low patient/dermatologist concordance of views on treatment outcomes. These findings also highlight a need to further incorporate the patient's views into treatment decision-making in Russia.

Funding: Novartis Pharma AG, Basel, Switzerland.

Keywords: Concordance between patient and dermatologist; Disease burden; Psoriasis; Quality of life; Real-world study; Treatment patterns

\section{INTRODUCTION}

Psoriasis is a chronic immune-mediated inflammatory condition that mainly affects the skin and occurs in $2-3 \%$ of the population worldwide $[1,2]$. The prevalence of psoriasis varies from $0.09 \%$ to $11.4 \%$ across countries depending upon the patients' age, region of residence and genetic factors [3]. Approximately 14 million people in Europe have psoriasis [4]. In 2015, the prevalence of psoriasis was noted as 233.4 per 100,000 population [5].

The most common form of psoriasis is plaque psoriasis (psoriasis vulgaris), in which patients may have sharply circumscribed, round-oval, or nummular (coin-sized) plaques. Chronic plaque-type psoriasis accounts for more than $90 \%$ of all cases [6]. It is typically found on the elbows, knees, scalp, and lower back. Psoriasis is usually classified as mild, moderate, or severe [2]. In approximately onethird of patients, the disease is characterised by a moderate-to-severe recurrent course. Both general and dermatology-specific quality of life (QoL) are reduced in psoriasis patients at each severity level from mild to severe [7]. Though the causes of psoriasis are not fully understood, well-recognised risk factors include family history and environmental risk factors such as smoking, stress, obesity, and alcohol consumption [8].

Itch (pruritus) is an important and common symptom in psoriasis, with approximately $60-85 \%$ of psoriatic patients suffering from itch [9-11]. The majority of patients with psoriasis consider itch to be a symptom that negatively affects their QoL, sleep and ability to work [12].

It is now recognised that psoriasis is not just a skin disease and has significant systemic effects. It is usually associated with an increased rate of comorbidities such as psoriatic arthritis (PsA), cardiovascular disease (CVD), type 2 diabetes (T2D), metabolic syndrome and obesity $[13,14]$. In addition to the physical impact of these conditions, psoriasis has a profound impact on the psychological wellbeing of the patient, with anxiety and depression being commonly diagnosed in patients with psoriasis [15]. These comorbidities limit the patient's ability to engage in the physical activities of day-to-day life [16].

Psoriasis patients have lower QoL due to physical symptoms such as itching, scaling and joint pain along with the economic and psychosocial impacts of the condition, which lead to problems with self-esteem, stigmatisation, and feelings of anger, embarrassment and shame according to the National Psoriasis Foundation [17].

The Federal Mandatory Medical Insurance Fund, as per the mandatory medical insurance (OMS), is the primary source of healthcare expenditure in Russia [18]. Healthcare delivery as well as prescription drugs are covered by state and regional lists of services, while their reimbursement rates are determined by the OMS. The European consensus on treatment goals has laid the foundation for optimising psoriasis care by standardising therapeutic decisions based on endpoint and outcome measures over defined periods of time, using a graded approach based on psoriasis severity $[19,20]$. As per the European guidelines, patients with mild psoriasis are mainly treated with topical agents. Ultraviolet (UV) phototherapy and traditional systemic medications/oral immunosuppressant (methotrexate, cyclosporine or retinoid) therapy alone or in combination with topical therapy are used for the treatment of moderate-to- 
severe psoriasis. Biologics are usually used only after other treatments have failed or if the patient has very severe symptoms [21]. Similarly, as per the Russian guidelines, mild psoriasis is treated with topical therapy (glucocorticoids, synthetic vitamin D3, salicylic acid, etc.) \pm UV phototherapy, while moderateto-severe psoriasis is treated with mono- or combination systemic therapy, including disease-modifying nonbiologics (e.g. methotrexate) or biologics [22].

The objective of this study was to describe the demographics, clinical characteristics, comorbidities and treatment patterns of systemic therapy eligible psoriasis patients in Russia as well as to assess the impact of psoriasis on the QoL and work productivity of the patients. We also evaluated patient/dermatologist concordance on severity, signs/symptoms and satisfaction with psoriasis treatment.

\section{METHODS}

\section{Data Source}

The Growth from Knowledge (GfK) Disease Atlas global real-world evidence programme is a syndicated, retrospective, cross-sectional survey conducted among 524 dermatologists and 3821 patients in nine countries [United Kingdom (UK), France, Germany, Italy, Spain, Russia, South Korea, Brazil and Mexico] between September 2015 and January 2016. This research was conducted as a market research survey in accordance with the 1998 Data Protection Act (UK) and British Healthcare Business Intelligence Association (BHBIA) legal and ethical guidelines for healthcare market research. The survey was designed, implemented and reported in compliance with the ethical principles laid down in the Declaration of Helsinki. Although ethical approval was not required, each participant provided their informed consent for the anonymised, aggregated reporting of research findings.

Dermatologists were recruited from a large representative sample. They were screened for their eligibility to participate in the survey based on their experience ( $>3$ years' experience in practice post-training), being personally responsible for initiating/making treatment decisions, and seeing at least ten psoriasis patients per month. Systemic therapy eligible patients were defined as those who currently have or have ever had moderate-to-severe psoriasis as reported by dermatologists and received a prescription treatment for their psoriasis (systemic agents or topical treatments) at the time of the survey. The GfK Disease Atlas survey captured patient data through online patient record forms completed by the dermatologists and a paper survey completed by patients at the end of the consultation, prior to leaving the premises. Here, we report the results from the Russian dataset only.

\section{Outcomes}

\section{Demographics and Disease Characteristics}

Data captured in the survey included the following: demographic parameters (age, gender); disease profile (duration of the disease, severity); body surface area (BSA\%); [Physician's Global Assessment/Investigator's Global Assessment (PGA/IGA) indices]; medical history (previous and current treatment regimens); current symptoms (e.g. itching, flaking, inflamed skin, skin pain, scaling); and comorbid conditions (PsA, CVD, obesity, T2D and anxiety or depression).

Psoriasis severity was evaluated via the absolute Psoriasis Area and Severity Index (PASI) score as reported by the dermatologist, and was categorized into clear/almost clear $(\mathrm{PASI} \leq 5)$, mild $(5<$ PASI $\leq 10)$, moderate $(10<$ PASI $\leq 20)$ or severe (PASI $>20$ ). As systemic treatments are indicated for moderate-to-severe patients, moderate and severe psoriasis cases were combined in the analysis into a 'moderateto-severe' category (PASI > 10).

\section{QoL and Work Productivity}

Patient-reported QoL was assessed using the Dermatology Life Quality Index (DLQI) [23]. The DLQI is a dermatology-specific measure of a patient's health-related QoL. Impairment of work and daily activities due to psoriasis was assessed using the Work Productivity and 
Activity Impairment (WPAI) questionnaire [24]. The WPAI assesses overall work impairment, work time missed (absenteeism), time impaired while at work (presenteeism) and activity impairment over the previous 7 days. Work productivity loss is expressed as a combination of absenteeism and presenteeism. Patients who reported "currently not in employment" provided data only for the activity impairment domain.

\section{Concordance Between the Patient and Dermatologist}

Patient/dermatologist concordance was defined as both the patient and dermatologist providing identical answers to the same survey question. Patient/dermatologist concordance was assessed on the following survey questions, which were asked to both the patient and the dermatologist:

1. Psoriasis severity, based on the 5-point IGA/ PGA scale (clear/almost clear-1 or 2; mild -3 ; moderate to severe -4 or 5 )

2. Absence or presence of symptoms (scaling, itching, inflamed skin, flaking, painful skin, joint pain, cracked skin, burning, onycholysis, nail deformation and bleeding)

3. Extent of itch and skin pain, on a scale from 1 (almost no itch/skin pain) to 10 (worst imaginable itch/skin pain)

4. Satisfaction with overall control of psoriasis achieved, on a scale from 1 (not at all satisfied) to 7 (completely satisfied)

\section{Statistical Analysis}

Descriptive statistics relating to patient demographics (age, gender), psoriasis characteristics [severity, symptoms (itch and skin pain), disease duration, medical history (previous and current treatment regimens)], comorbidities and patient-reported outcomes (PROs-DLQI and WPAI) were reported for all patients and for patients categorised by psoriasis severity (clear/ almost clear, mild, moderate and severe, as per PASI score). We also examined the DLQI and WPAI for patients with specific comorbidities (PsA, CVD, obesity, T2D and anxiety or depression).
We calculated Cohen's $\kappa$ to assess concordance between patients and their dermatologists regarding disease severity, signs/symptoms and satisfaction with the level of disease control achieved. Kappa $(\kappa)$ values range between 0 (no agreement/concordance) and 1 (perfect agreement/concordance) [25]. Concordance was categorised based on the $\kappa$ value as follows: none $(\kappa \leq 0)$, none to slight $(0.01-0.20)$, fair (0.21-0.40), moderate $(0.41-0.60)$, substantial $(0.61-0.80)$ and almost perfect $(>0.8)$.

All statistical analyses were conducted using SPSS $^{\circledR}$ Statistics v22.0 (IBM Corp, Armonk, NY, USA) and R (https://www.r-project.org/).

\section{RESULTS}

\section{Demographics and Disease Characteristics}

Out of the 3821 systemic therapy eligible patients included in the survey, 300 were from Russia. Of those, $53 \%$ were male. The mean age of the Russian patients was 43.6 years [standard deviation $(\mathrm{SD}) \pm 14.3$ ], and their mean time since psoriasis diagnosis was 9.9 years $( \pm 11.2)$. Psoriasis severity at the time of the survey ranged from clear to severe despite current treatment, with a mean absolute PASI of $9.0( \pm 7.6)$. Compared to those with mild psoriasis, patients with severe psoriasis were older (52.1 years \pm 12.6 years vs. 42.7 years \pm 13.1 years; $p=0.003$ ) and had longer disease durations 18.0 years \pm 14.8 years vs. 9.9 years \pm 11.6 years; $p=0.001$ ) (Table 1).

Patients with clear/almost clear, mild or moderate psoriasis tended to be younger than those with severe psoriasis $(p=0.097)$. For example, the proportion of patients $<45$ years of age who had clear/almost clear to moderate psoriasis was $47-56 \%$, whereas the corresponding proportion with severe psoriasis was only $28 \%$. Among the systemic therapy eligible patients enrolled in this survey, the majority (67\%) currently had psoriasis on visible and nonvisible areas, and $43 \%$ of the patients had psoriasis in sensitive and nonsensitive areas (Table 1). 
Table 1 Demographics and disease characteristics by psoriasis severity at the time of the survey

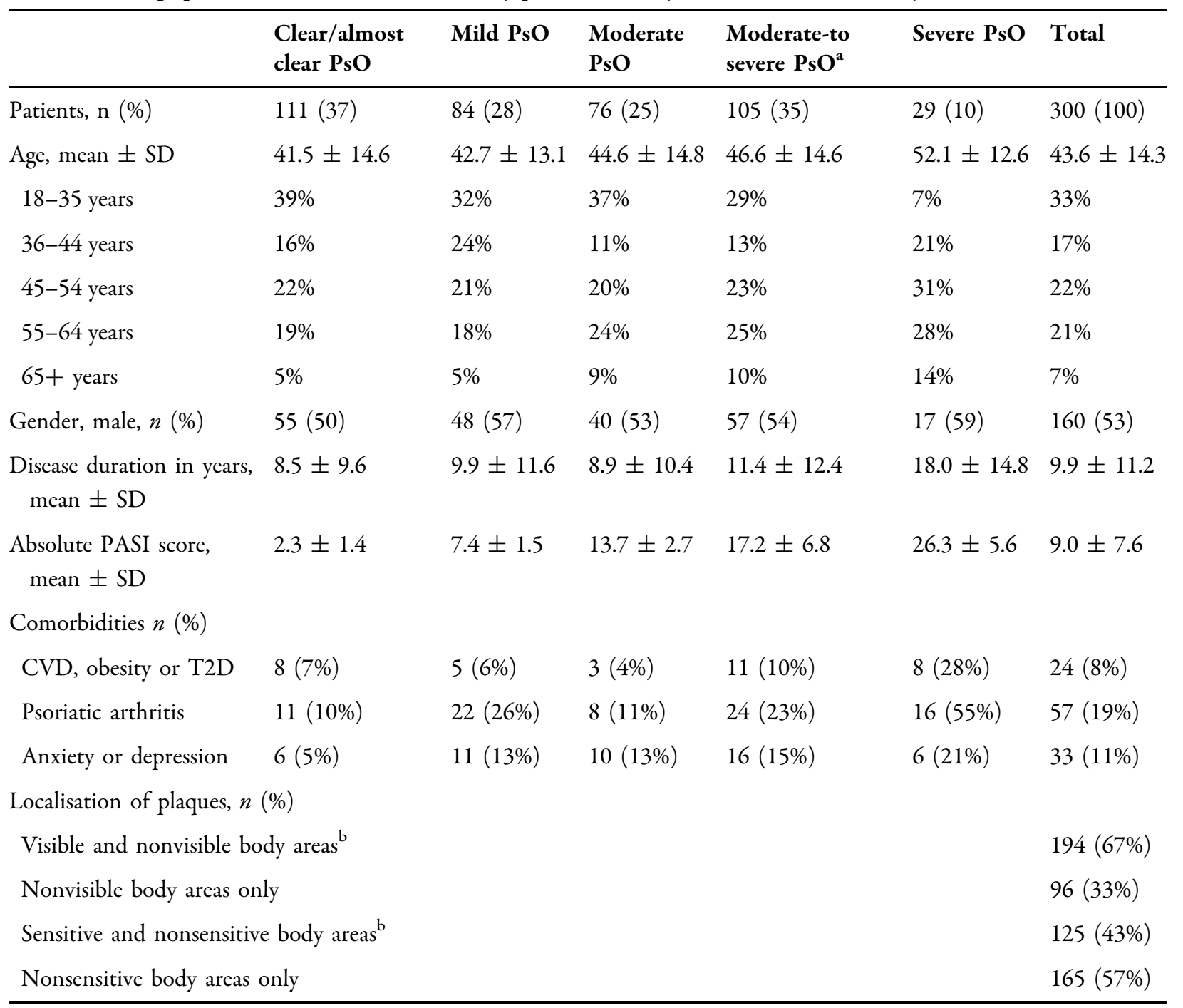

$C V D$ cardiovascular disease, DLQI Dermatology Life Quality Index, PASI Psoriasis Area and Severity Index, PsO psoriasis, $S D$ standard deviation, $T 2 D$ type 2 diabetes

a Moderate and severe cases were combined in the 'moderate to severe' category

b Visible body areas were defined as body areas not covered by clothing (scalp, face, back of hands, palms of hands, fingers, nails) and therefore visible to the patient's entourage; sensitive body areas were defined as body areas where the skin is thinner and may be more sensitive to treatment (genitals, scalp, face). Based on Psoriasis Association definitions [26] and data available in the survey

\section{Itch/Skin Pain}

Overall, according to dermatologists, $51 \%$ of patients suffered from itch and 25\% from skin pain. The proportion of patients with itch and skin pain increased with current psoriasis severity ( $p=0.000)$ (Fig. 1$)$. Itch and skin pain were also reported to be some of the most bothersome symptoms in these psoriasis patients $[23 \%$ (64 patients) and $10 \%$ (28 patients), respectively] (data not shown). 


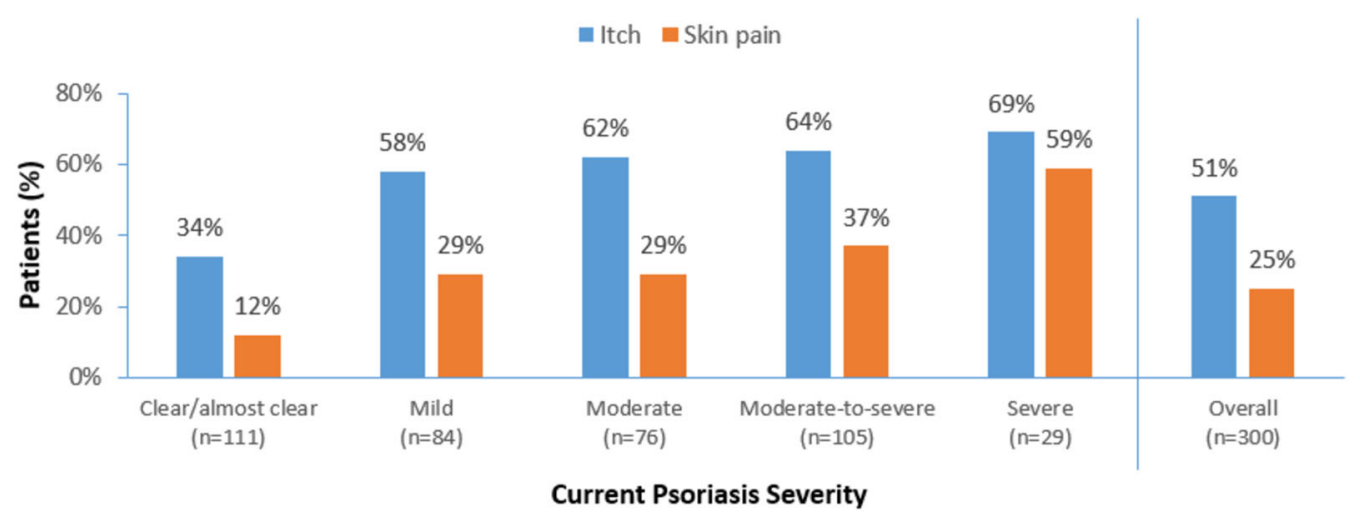

Fig. 1 Itch and skin pain in psoriasis patients categorised by psoriasis severity at the time of the survey. Dermatologists were asked whether their patient experienced itch at the time of the survey, and to assess itch extent on a scale

\section{Physical and Psychological Comorbidities}

Among the total study population, 8\% $(n=24)$ of the patients had CVD, obesity or T2D as a comorbidity, $19 \%(n=57)$ pad PsA and $11 \%$ $(n=33)$ had anxiety or depression (Table 1$)$. The presence of comorbidities increased with disease severity despite the small sample size (data not shown).

\section{Quality of Life}

Mean DLQI score was $7.1( \pm 6.0)$, corresponding to a moderate effect of the skin disease on patient QoL. Overall, the more severe the disease, the greater the impact on patient QoL ( $p$ $=0.000$ ) (Fig. 2). Overall, all types of comorbidities were associated with a poorer QoL, with the greatest impact observed in obese psoriasis patients; there was a difference of 6.9 between obese and non-obese patients $(p=0.000)$ (Fig. 3).

\section{Work Productivity}

Amongst employed patients, the mean work productivity impairment was 33.2\% ( \pm 26.8 ). Mean work absenteeism due to psoriasis was $8.6 \% \quad( \pm 19.0)$, mean impairment while working (presenteeism) was 27.9\% $( \pm 22.8)$ from 0 (no itch) to 10 (worst imaginable itch). The relationship between disease severity and percentage of people with symptoms was statistically significant $(p=0.000)$

and mean activity impairment was $36.2 \%$ $( \pm 25.7)$. There was a trend for greater work impairment with more severe disease $(p=$ 0.005) (Fig. 4).

The presence of comorbidities was associated with higher overall work impairment in psoriasis patients (except in patients with T2D). The greatest work impairment was observed in patients with obesity $(45 \% \pm 27.8 \%)$ followed by patients with anxiety or depression $(44.3 \% \pm 34.0), \quad$ CVD $\quad(43.4 \% \pm 39.6), \quad$ PsA $(38.3 \% \pm 27.5)$ and $\mathrm{T} 2 \mathrm{D}(26.9 \% \pm 23.9)$ (data not shown).

\section{Treatment Patterns}

The majority of the systemic therapy eligible psoriasis patients in this survey were treated with topical agents only (60\%), followed by a combination of conventional systemic and topical agents (33\%). No more than $2 \%$ of the patients received biologic treatments [among these, infliximab and ustekinumab were used in $83 \%$ and $17 \%$ of the cases, respectively (data not shown)]. With increasing psoriasis severity, the proportion of patients who were only on only topical agents reduced; this proportion was $62 \%$ in patients with mild psoriasis compared with only $35 \%$ in those with severe psoriasis (Fig. 5). 


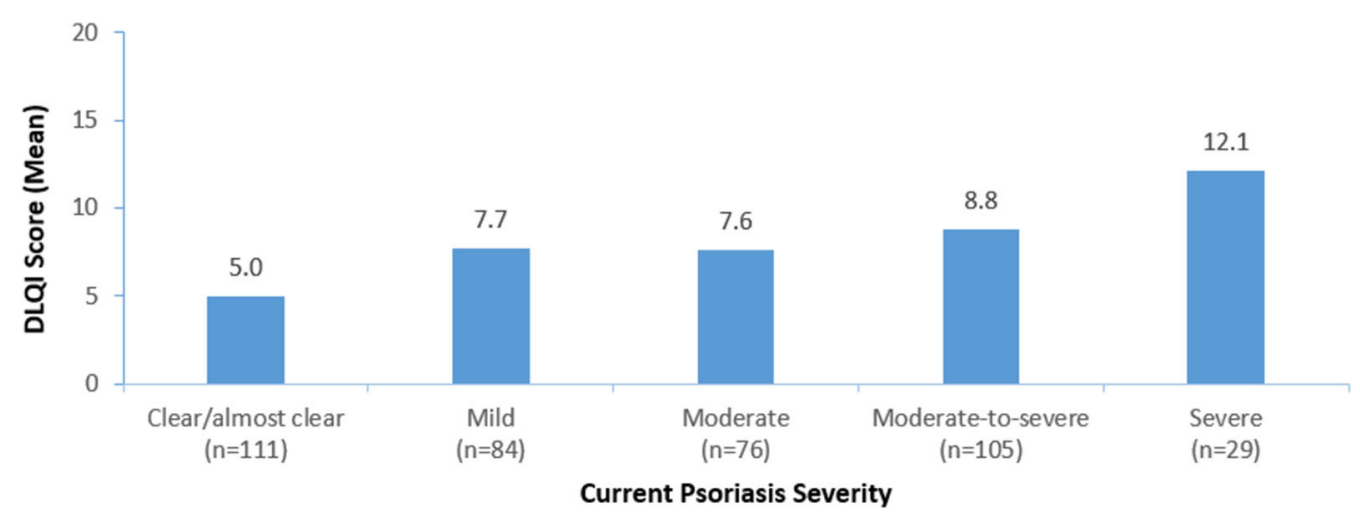

Fig. 2 Quality of life in psoriasis patients according to disease severity. Dermatology Life Quality Index (DLQI) scores range from 0 to 30 ; higher scores indicate lower QoL $(0-1=$ no effect on the patient's life; $2-5=$ small

effect; $\quad 6-10=$ moderate effect; $11-20 / 21-30=$ very large/extremely large effect). The relationship between disease severity and DLQI score was statistically significant $(p=0.000)$

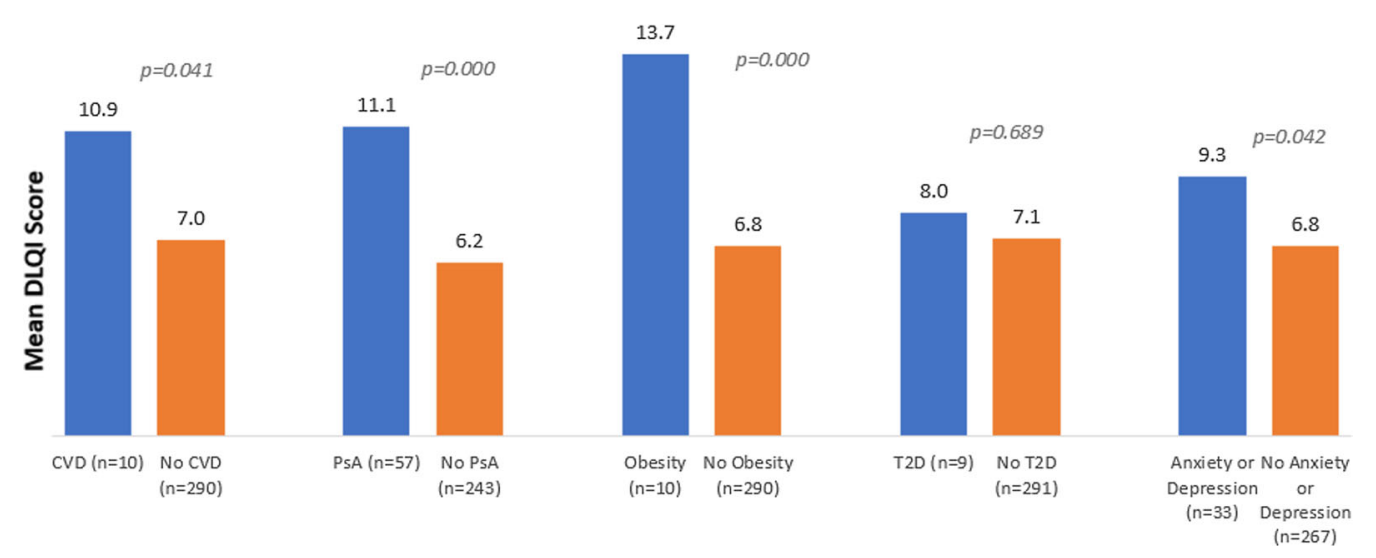

Fig. 3 QoL of psoriasis patients with comorbidities. Dermatology Life Quality Index (DLQI) scores range from 0 to 30; higher scores indicate lower quality of life (QoL). CVD cardiovascular disease, $P s A$ psoriatic arthritis, T2D type 2 diabetes

\section{Concordance Between Dermatologists and Their Psoriasis Patients on Disease Severity, Signs/Symptoms and Satisfaction with Disease Control Achieved}

Fair patient/dermatologist concordance was observed for psoriasis severity $(\kappa=0.320)$, and $58 \%$ of the patients and their dermatologists were in agreement regarding the current severity of the disease (Fig. 6). Concordance on the presence of symptoms ranged from fair to substantial, with the lowest and highest concordance observed for scaling $(69 \%$ agreement, $\kappa=0.356)$ and joint pain $(93 \%$ agreement, $\kappa=0.714)$, respectively. The lowest concordance between dermatologists and their patients was seen when assessing the extent of itch (34\% agreement; $\kappa=0.261)$ and of skin pain $(31 \%$ agreement; $\kappa=0.253)$. There was only slight concordance between dermatologists and their patients $(34 \%$ agreement; $\kappa=0.160$ ) on satisfaction with the overall disease control achieved.

\section{DISCUSSION}

This patient-dermatologist real-world survey provides insight into the current psoriasis treatment patterns and the impact of psoriasis on QoL and work productivity among systemic therapy eligible psoriasis patients in Russia. 
Work productivity loss (\% overall work impairment due to psoriasis)

absenteeism (\% work time missed due to psoriasis)

॥- Presenteeism (\% impairment while working due to psoriasis)

= Activity impairment (\% activity impairment due to psoriasis)

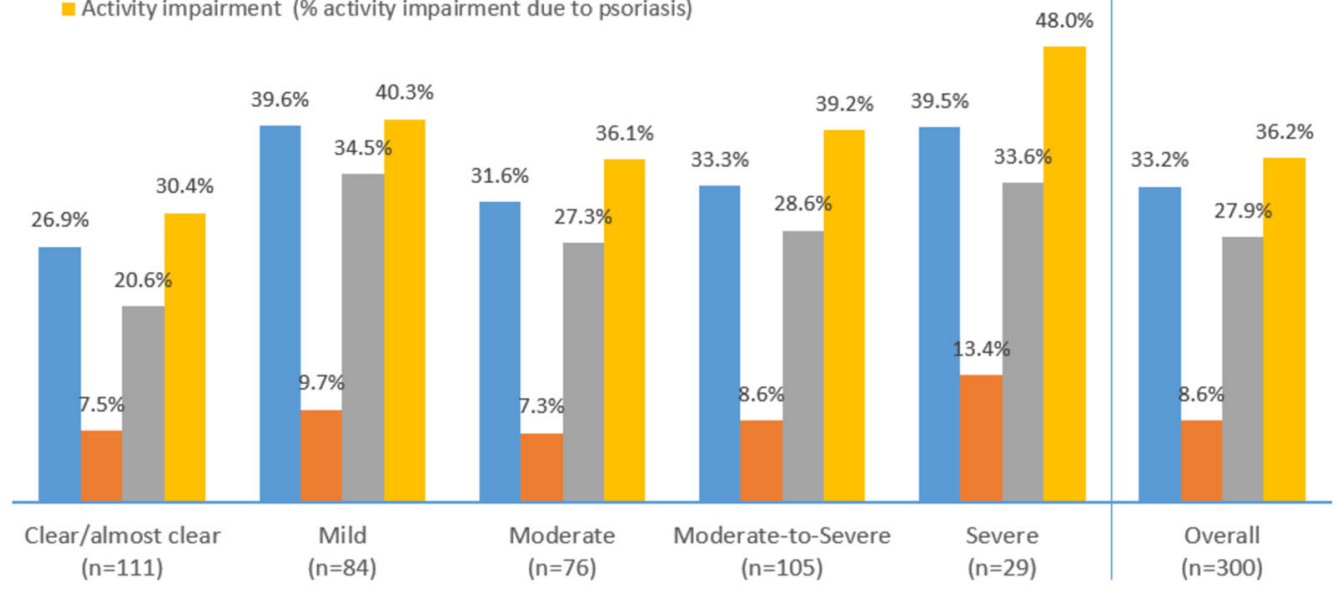

Current Psoriasis Severity

Fig. 4 Work productivity loss and activity impairment in psoriasis patients categorised by psoriasis severity at the time of the survey. Work Productivity and Activity Impairment (WPAI) scores range from 0 to $100 \%$; higher scores indicate greater impairment and less productivity. The relationship between disease severity and work productivity was statistically significant $(p=0.005)$

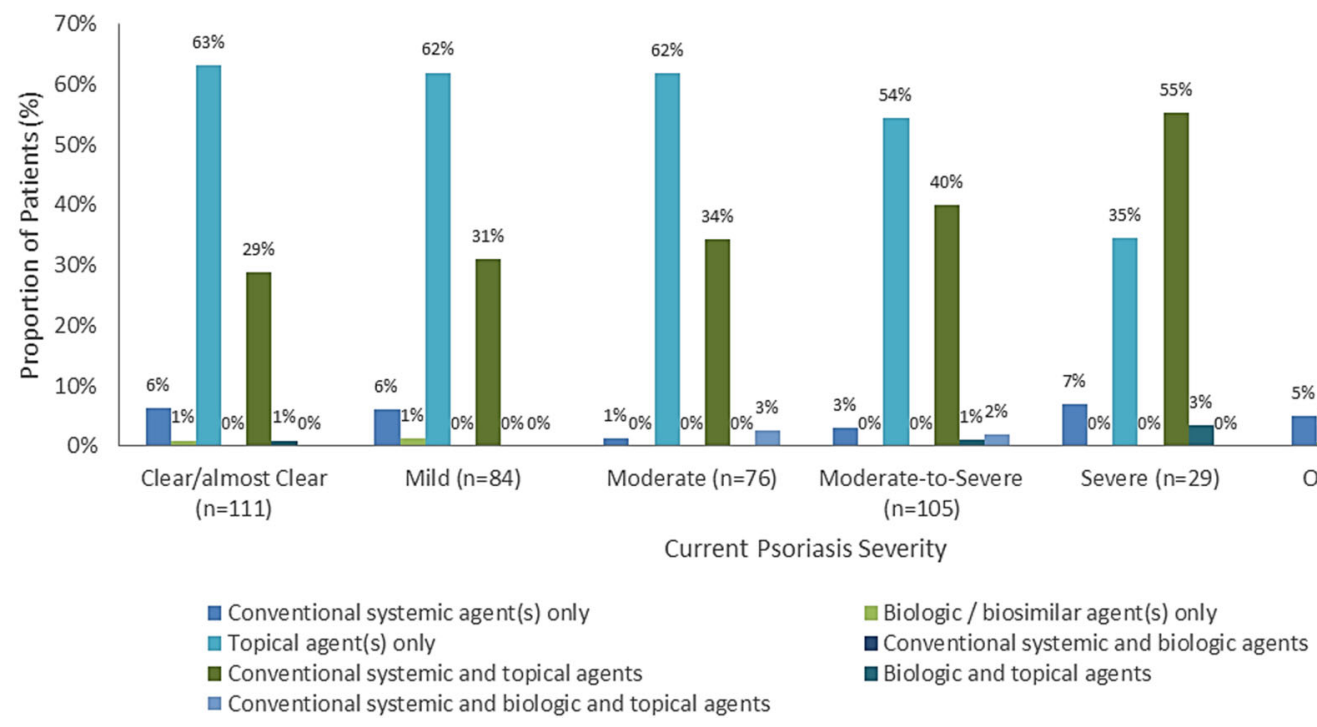

Fig. 5 Therapeutic approaches to psoriasis used in the Russian sample. The seven mutually exclusive therapy subgroups account for $100 \%$ of the patients. The

We observed a high prevalence of moderateto-severe psoriasis among young adults, though severe psoriasis was higher in patients above 45 years of age. Having severe disease or remaining treatment regimens are included in these seven subgroups. OTC over the counter

comorbidities (physical and psychological) further increased the QoL and work productivity burden on these patients. There was a trend for greater work productivity impairment with 


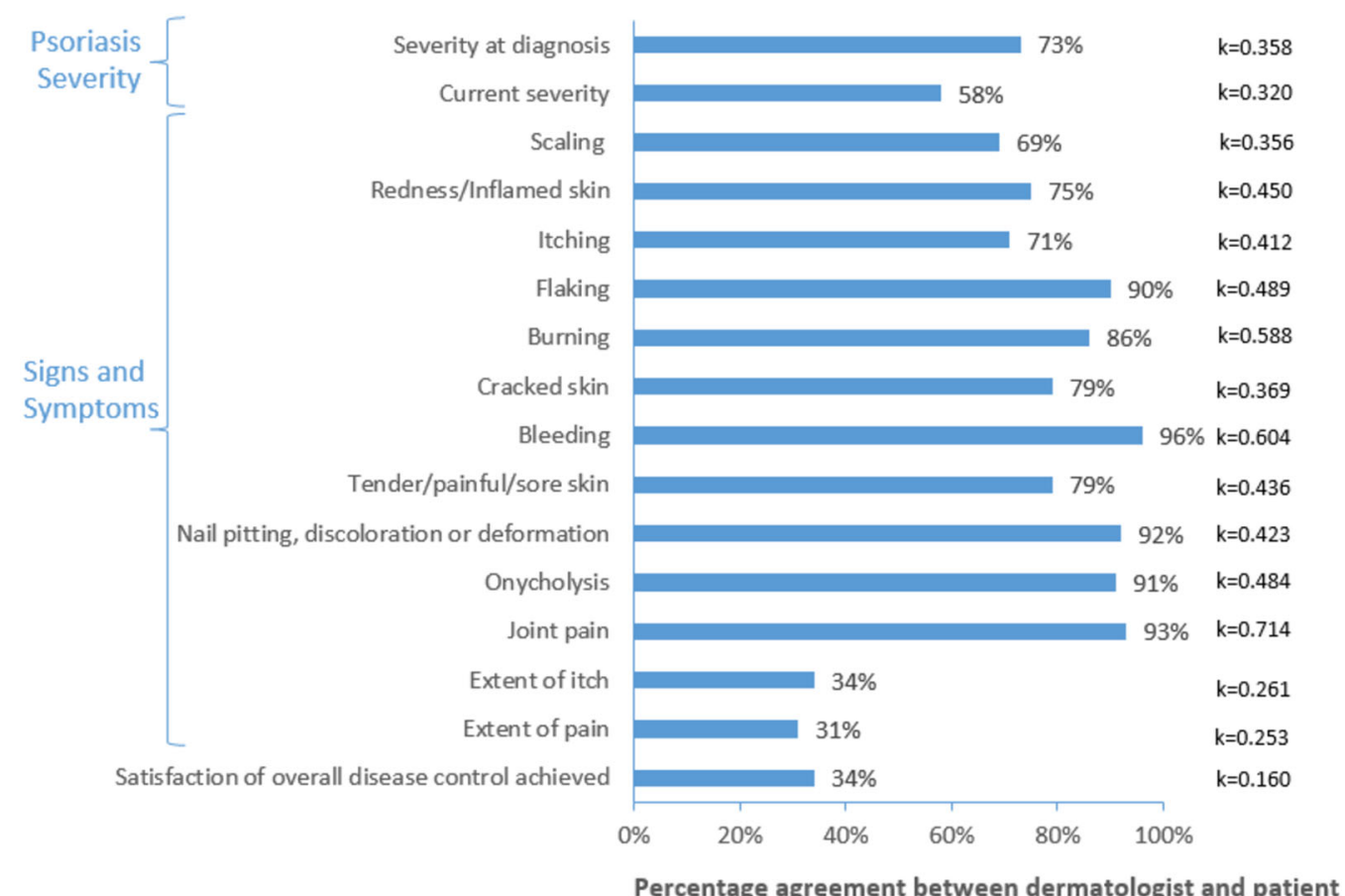

Fig. 6 Concordance between dermatologist and patient assessments of psoriasis severity, presence of signs/symptoms and satisfaction with the disease control achieved. Cohen's kappa $(\kappa)$ values range between 0 (no agreement/concordance) and 1 (perfect agreement/concordance). Levels of

increasing disease severity. However, due to the small sample size, we conducted only descriptive analyses, and it is difficult to explain why the work impairment was comparatively high among patients with mild psoriasis. Overall, the findings from this survey are consistent with results from other studies signifying a great disease burden on psoriasis patients, comparable to the disability caused by other major medical conditions [25-27]. It is worth noting that a significant presence of symptoms, reduced QoL and work impairment was evident in all the enrolled patients, even though they were being treated for their psoriasis at the time of the survey.

We also observed that the treatment practice for psoriasis patients in Russia was mostly based on topical therapy (used in 60\% of the cases) and combination therapy with conventional systemic and topical agents (in 33\% of patients). Though the introduction of cost-effective biologics has significantly changed the psoriasis concordance were defined based on the value of $\kappa$ as follows: $\leq 0$ : none; $0.01-0.20$ : none to slight; $0.21-0.40$ : fair; 0.41-0.60: moderate; $0.61-0.80$ : substantial; $>0.80$ : almost perfect

treatment paradigm in the last few years $[28,29]$, biologics or their biosimilars were prescribed to only a very small proportion of patients (2\%) in this survey.

Moreover, despite the patients receiving a prescribed treatment for their psoriasis as per the study enrolment criteria, over one-third of patients still had moderate-to-severe psoriasis, suggesting an unmet treatment need. The level of agreement between dermatologist and patient satisfaction with the overall disease control achieved was fairly low: only $34 \%$ of the patients and their dermatologists had the same level of satisfaction with treatment. Also, the level of agreement between the patient and the dermatologist was low in their assessments of the extent of itch and skin pain, with only $34 \%$ and $31 \%$ of the patients and their dermatologists in agreement, respectively. This suggests a lack of awareness or understanding among dermatologists of the patient's views on their disease, how psoriasis affects their daily living, 
and their experience with previous treatments. Thus, a successful treatment regimen should incorporate the patient's perspective into treatment decision-making, as advocated by several other studies [30, 31].

Results from the complete GfK Disease Atlas survey have been published $[32,33]$. The finding that disease burden on psoriasis patients is affected by physical as well as psychological comorbidities remains true for the Russian as well as the larger multinational cohort [32]. Comparisons of countries showed that disease severity and disease control related patient-dermatologist concordance in Russia was among the lowest of all the countries included in the survey [33].

There are some limitations of this study, including low sample size, descriptive analyses, the lack of a statistical control for potential confounding variables and an inability to establish causal relationships between psoriasis and related comorbidities. The cross-sectional design of this study did not permit an investigation of the changes in PROs over time relative to the changes in disease severity. Generic PROs such as WPAI may not be sensitive enough to detect differences between different groups of patients. Also, it is difficult to compare and interpret the results of this survey in the absence of similar data from Russian patients obtained in other studies. Thus, further research based on longitudinal data will allow for a better understanding of the dynamics in Russian psoriasis patients.

\section{CONCLUSIONS}

The results of this study demonstrate a substantial disease burden on systemic therapy eligible psoriasis patients, despite receiving treatment for their psoriasis. Considering that psoriasis is a lifelong disease and can affect all aspects of a patient's life, even in patients with limited skin involvement, a successful treatment plan should address both the physical and psychological aspects of psoriasis. Thus, understanding the patient's views and incorporating them into treatment decision-making are important for the effective management of psoriasis. The patient's perspective needs to be further explored, as it may differ considerably from the dermatologist's perspective.

\section{ACKNOWLEDGEMENTS}

The authors would like to thank the patients and the dermatologists who participated in the survey.

Funding. This study was funded by Novartis Pharma AG, Basel, Switzerland. Article processing charges were funded by Novartis Pharma AG, Basel, Switzerland. The sponsor had no influence on the evaluation of data. All authors had full access to all the data in this study and take complete responsibility for the integrity of the data and accuracy of the data analysis.

Medical Writing. The authors acknowledge Kavita Rodha (Novartis Healthcare Pvt. Ltd., Hyderabad, India) for writing/editorial assistance with this manuscript.

Authorship. All named authors meet the International Committee of Medical Journal Editors (ICMJE) criteria for authorship for this manuscript, take responsibility for the integrity of the work as a whole, and have given final approval to the version to be published.

Disclosures. Alexey Kubanov is a full-time employee of the State Research Center of Dermatology and Venerology of the Russian Ministry of Health, Moscow, Russia. Andrey Bakulev is a full-time employee of the State Medical University of the Ministry of Health, Saratov, Russia. Tatiana Fitileva is a full-time employee of Novartis Pharma, LLC, Moscow, Russia. Evgenia Novoderezhkina is a full-time employee of Novartis Pharma, LLC, Moscow, Russia. Isabelle Gilloteau is a full-time employee of Novartis Pharma AG, Basel, Switzerland. Haijun Tian is a full-time employee of Novartis Pharmaceuticals Corporation, East Hanover, United States. Tanya Howe is a full-time employee of GfK, London, UK. Guilhem Pietri is a consultant for GfK, London, UK. 
Compliance with Ethics Guidelines. This research was conducted as a market research survey in accordance with the 1998 Data Protection Act (UK) and British Healthcare Business Intelligence Association (BHBIA) legal and ethical guidelines for healthcare market research. The survey was designed, implemented and reported in compliance with the ethical principles laid down in the Declaration of Helsinki. Although ethical approval was not required, each participant provided their informed consent for the anonymised, aggregated reporting of research findings.

Data Availability. The datasets generated during and/or analyzed during the current study are available from the corresponding author on reasonable request.

Open Access. This article is distributed under the terms of the Creative Commons Attribution-NonCommercial 4.0 International License (http://creativecommons.org/licenses/ by-nc/4.0/), which permits any noncommercial use, distribution, and reproduction in any medium, provided you give appropriate credit to the original author(s) and the source, provide a link to the Creative Commons license, and indicate if changes were made.

\section{REFERENCES}

1. Glickman FS. Lepra, psora, psoriasis. J Am Acad Dermatol. 1986;14(5 Pt 1):863-6.

2. Pariser DM, Bagel J, Gelfand JM, Korman NJ, Ritchlin CT, Strober BE, et al. National Psoriasis Foundation clinical consensus on disease severity. Arch Dermatol. 2007;143:239-42.

3. WHO. Global report on psoriasis. 2016. http://apps. who.int/iris/bitstream/10665/204417/1/97892415651 89_eng.pdf. Accessed 27 Nov 2016.

4. Ortonne JP, Prinz JC. Alefacept: a novel and selective biologic agent for the treatment of chronic plaque psoriasis. Eur J Dermatol. 2004;14(1):41-5.

5. Kubanova AA, Kubanov AA, Melechina LE, Bogdanova EB. Organization of medical care on the profile of "dermatovenereology" in the Russian Federation. The dynamics of the incidence of infections, sexually transmitted diseases, skin diseases and subcutaneous tissue, 2013-2015. Vestn Dermatol Venerol. 2016;3:12-28.

6. Griffiths CE, Barker JN. Pathogenesis and clinical features of psoriasis. Lancet. 2007;370(9583):263-71.

7. Korman NJ, Zhao Y, Pike J, Roberts J. Relationship between psoriasis severity, clinical symptoms, quality of life and work productivity among patients in the USA. Clin Exp Dermatol. 2016;41:514-21.

8. Huerta C, Rivero E, Garcia Rodriguez LA. Incidence and risk factors for psoriasis in the general population. Arch Dermatol. 2007;143(12):1559-65.

9. Sampogna F, Gisondi P, Melchi CF, et al. Prevalence of symptoms experienced by patients with different clinical types of psoriasis. $\mathrm{Br} \mathrm{J}$ Dermatol. 2004;151(3):594-9.

10. Yosipovitch G, Goon A, Wee J, Chan YH, Goh CL. The prevalence and clinical characteristics of pruritus among patients with extensive psoriasis. $\mathrm{Br} \mathrm{J}$ Dermatol. 2000;143(5):969-73.

11. Bakulev AL, Shagova YV, Kozlova IB. Psoriasis as a systemic disease. Saratov J Med Sci Res. 2008;4(1):13-20.

12. Amatya B, Wennersten G, Nordlind K. Patients' perspective of pruritus in chronic plaque psoriasis: a questionnaire-based study. J Eur Acad Dermatol Venereol. 2008;22(7):822-6.

13. Gottlieb AB, Chao C, Dann F. Psoriasis comorbidities. J Dermatol Treat. 2008;19(1):5-21.

14. Yeung H, Takeshita J, Mehta NN, et al. Psoriasis severity and the prevalence of major medical comorbidity: a population-based study. JAMA Dermatol. 2013;149(10):1173-9.

15. Kurd SK, Troxel AB, Crits-Christoph P, Gelfand JM. The risk of depression, anxiety, and suicidality in patients with psoriasis: a population-based cohort study. Arch Dermatol. 2010;146(8):891-5.

16. Helmick CG, Lee-Han H, Hirsch SC, Baird TL, Bartlett CL. Prevalence of psoriasis among adults in the U.S.: 2003-2006 and 2009-2010 National Health and Nutrition Examination Surveys. Am J Prev Med. 2014;47(1):37-45.

17. Armstrong AW, et al. Quality of life and work productivity impairment among psoriasis patients: findings from the National Psoriasis Foundation survey data 2003-2011. PLoS One. 2012;7(12):e52935. 
18. Popovich L, Potapchik E, Shishkin S, Richardson E, Vacroux A, Mathivet B. Russian Federation: health system review. Health Syst Transit. 2011;13(7):1-190.

19. Reich K, Mrowietz U. Treatment goals in psoriasis. J Dtsch Dermatol Ges. 2007;5(7):566-74.

20. Mrowietz U, Kragballe K, Reich K, et al. Definition of treatment goals for moderate to severe psoriasis: a European consensus. Arch Dermatol Res. 2011;303(1):1-10.

21. Nast A, Gisondi P, Ormerod AD, et al. European S3guidelines on the systemic treatment of psoriasis vulgaris: update 2015. EDF in cooperation with EADV and IPC. 2015. http://www.euroderm.org/ edf/index.php/edf-guidelines/category/5-guidelinesmiscellaneous. Accessed 1 Aug 2017.

22. Kubanova AA, editor. Federal clinical guidelines. Dermatovenereology 2015: diseases of the skin, sexually transmitted infections. 5th ed. Moscow: Delovoi Express; 2016.

23. Finlay AY, Khan GK. Dermatology Life Quality Index (DLQI) - a simple practical measure for routine clinical use. Clin Exp Dermatol. 1994;19(3):210-6.

24. Reilly MC, Zbrozek AS, Dukes EM. The validity and reproducibility of a work productivity and activity impairment instrument. Pharmacoeconomics. 1993;4(5):353-65.

25. Finlay AY, Coles EC. The effect of severe psoriasis on the quality of life of 369 patients. Br J Dermatol. 1995;132(2):236-44.

26. Rapp SR, Feldman SR, Exum L, et al. Psoriasis causes as much disability as other major medical diseases. J Am Acad Dermatol. 1999;41(3 Pt 1):401-7.
27. Stern RS, Nijsten T, Feldman SR, et al. Psoriasis is common, carries a substantial burden even when not extensive, and is associated with widespread treatment dissatisfaction. J Investig Dermatol Symp Proc. 2004;9(2):136-9.

28. Ahn CS, Gustafson CJ, Sandoval LF, Davis SA, Feldman SR. Cost effectiveness of biologic therapies for plaque psoriasis. Am J Clin Dermatol. 2013;14(4):315-26.

29. Anis AH, Bansback N, Sizto S, Gupta SR, Willian MK, Feldman SR. Economic evaluation of biologic therapies for the treatment of moderate to severe psoriasis in the United States. J Dermatolog Treat. 2011;22(2):65-74.

30. Feuerhahn J, Blome C, Radtke MA, Augustin M. Validation of the patient benefit index for the assessment of patient-relevant benefit in the treatment of psoriasis. Arch Dermatol Res. 2012;304(6):433-41.

31. Lebwohl MG, Bachelez H, Barker J, et al. Patient perspectives in the management of psoriasis: results from the population-based Multinational Assessment of Psoriasis and Psoriatic Arthritis Survey. J Am Acad Dermatol. 2014;70(5):871-81.e1-30.

32. Griffiths C, Jo S, Naldi L, et al. A multidimensional assessment of the burden of psoriasis: results from a multinational dermatologist and patient survey. $\mathrm{Br}$ J Dermatol. 2018;179:173-81.

33. Griffiths C, Augustin M, Naldi L, et al. Patient-dermatologist agreement in psoriasis severity, symptoms and satisfaction: results from a real-world multinational survey. J Eur Acad Dermatol Venereol. 2018;32(9):1523-9. https://doi. org/10.1111/jdv.14937. 